\title{
Khayyam; progress and prospects of coupling a Spatial Heterodyne Spectrometer (SHS) to a Cassegrain Telescope for Optical interferometry
}

\author{
Sona Hosseini ${ }^{\mathrm{a}}$, Walter Harris ${ }^{\mathrm{b}}$, \\ ${ }^{a}$ Jet Propulsion Laboratory, California Institute of Technology, Pasadena, California 91109, USA \\ bunar and Planetary Laboratory, University of Arizona, Tucson, AZ 85721, USA
}

\begin{abstract}
In the temporal study of faint, extended sources at high resolving power, Spatial Heterodyne Spectrometer (SHS) can offer significant advantages about conventional dispersive grating spectrometers. We describe here a four-year continuous progress in Mt. Hamilton, Lick Observatory, toward development of a prototype reflective Spacial Heterodyne Spectrometer, Khayyam, instrument-telescope configuration to combine all of the capabilities necessary to obtain high resolving power visible band spectra of diffuse targets from small aperture on-axis telescopes where significant observing time can be obtained. We will discuss the design considerations going into this new system, installation, testing of the interferometer-telescope combination, the technical challenges and procedures moving forward.
\end{abstract}

Keywords: Tunable Spatial Heterodyne Spectrometer, SHS, extended targets, interferometry, spectrometry,

\section{INTRODUCTION}

Spectroscopy is a staple technique for remote sensing of astrophysical targets, but the level of detail obtained is limited by sensitivity, the spatial extent of the target, and the resolving power (R) of the instrument. The most common used instruments to obtain high resolving power spectra are the dispersive grating spectrometers at large aperture telescopes which compensate for their very small field of view (FOV) and under sample extended targets [1-3]. And because large aperture telescopes tend to be over-subscribed their usefulness is limited for temporal monitoring. Furthermore, the instrumental reliance on telescope aperture to provide étendue for these instruments essentially eliminates such instruments from space applications and in situ probes.

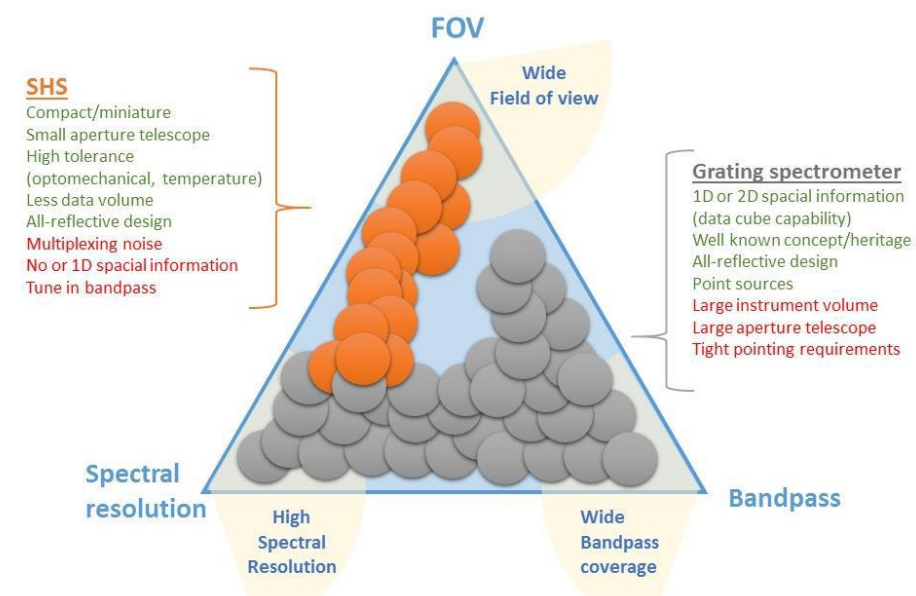

Figure 1. Conventional spectrometers are optimized to obtain high spectral resolution spectra from point sources and small FOVs at the wide spectral region. SHS technology can achieve high spectral resolution spectra over wide FOV using a small spectral bandpass of a target wavelength region.

Optical and Infrared Interferometry and Imaging V, edited by Fabien Malbet,

Michelle J. Creech-Eakman, Peter G. Tuthill, Proc. of SPIE Vol. 9907, 99072

(C) 2016 SPIE · CCC code: $0277-786 X / 16 / \$ 18 \cdot$ doi: $10.1117 / 12.2233120$ 
In the temporal study of faint, extended sources at high resolving power, Spatial Heterodyne Spectrometer (SHS) can offer significant advantages about conventional dispersive grating spectrometers [4, 5]. SHS is a miniature, all-reflective twobeam cyclical interferometer that can observe targeted atomic and molecular gas spectral lines at high spectral resolution. It comprises a grating and reflective optics tailored to a target wavelength region (UV to IR) with a solid-state array detector that produces optical interference fringes, whose Fourier transform produces high-resolution spectra. With its high optical throughput (étendue) and wide field of view (FOV), it has high sensitivity to weak or diffuse sources such as comet coma and Earth atmosphere gases [5, 6]. SHS shares some similarities with the traditional Fabry-Perot interferometer but offers a simpler approach to an optical geometry readily made compact and robust to alignment and a high tolerance to environmental optomechanical and temperature variations. SHS is good for survey observations for which the traditional Fourier transform interferometers (FTIR) capture a wide wavelength region of many gases and absorption bands, albeit with small FOV.

Khayyam was initially developed at the University of California, Davis (UCD) with the hope to perform nightly observation from the UCD campus coupled to an off-axis roof-top heliostats [7, 8]. The core of Khayyam is a tunable allreflective spatial heterodyne spectrometer (TSHS) and has up to 50 arcsec input field of view, resolving power up to 176000 , and a tunable bandpass covering $400 \mathrm{~nm}>\Delta \lambda_{B}>700 \mathrm{~nm}$ [9]. The heliostat was designed to be installed on top of the roof of the UCD laboratory at second floor. Using the two computer programmed rotating flat mirrors, the light was guided into the lab on the optical table where Khayyam was setup. With the extended delivery delay in the UCD heliostat system, in late 2010, Khayyam was transferred to Mt. Hamilton, a University of California observatory facility located in East of San Jose, CA, for the field test. The goal of the project was to study spatially extended astronomical targets where high resolving power is necessary to separate faint spectral signals, crowded bands, and to sample low $(<10 \mathrm{~km} / \mathrm{s})$ velocities at rapid temporal cadence. Over the next four-years, a continuous progress was made toward development of a prototype reflective SHS instrument-telescope configuration to combine all of the capabilities necessary to obtain high resolving power visible band spectra of diffuse targets from small aperture on-axis telescopes where significant observing time can be obtained. The majority of the effort was put into mounting Khayyam, to the fixed focal plane shared by the 0.6m Coudé Auxiliary Telescope (CAT) and the $3 \mathrm{~m}$ Shane Telescope and the successful alignment process with the CAT after eliminating the environmental effects such as vibrations. Many target data was taken by Khayyam, bot unambiguous scientific results was not feasible due to the coupling telescope configuration: both CAT and Shane telescopes contain supporting spider pattern for their secondary mirrors in collimated light and the preliminary data of Khay yam show the spider pattern appears in the fringe localization plane (FLP) as described latter in this paper.

Our present effort involves a full description of the ongoing development toward calibration process, improving the data reduction pipeline and investigating the experiments and effects of coupling SHS to a Cassegrain telescope. We will discuss the design considerations going into this new system, installation, testing of the interferometer-telescope combination, the technical challenges and procedures moving forward.

\section{TUNABLE REFLECTIVE SHS CONCEPT}

A reflective Special Heterodyne Spectrometer (SHS) is a compact two-beam interferometer which the input spectrum can be obtained via a Fourier transform. The dispersion is into symmetric orders, with the two beams counter circulating through a series of mirrors before returning to the grating and exiting the system (figure 2).

In reflective SHS (figure 2) the grating diffracts an incoming collimated beam into $\pm m$ orders following the grating equation for normal incidence $\left[\sin \beta_{\text {in }}+\sin \beta_{\text {out }}\right] \cos \Phi=m \lambda G$, where $\lambda$ is the wavelength, $\beta_{\text {in }}$ and $\beta_{\text {out }}$ are the incoming and outgoing angles, $\Phi$ is the angle between the incoming beams relative to the grating normal perpendicular to the diffraction plane, $G$ is the groove density. The incoming light beam hits the grating in the normal incidence and splits to two diffraction order $(m= \pm l)$. Both orders anti-symmetrically traverse the optical path, converge back on the grating, and diffract for the second time before exiting the system. For the tuned wavelength $\lambda$, called the heterodyne wavelength, the optics are aligned in such a way to have both orders exit the grating in normal angle: their wave-fronts exit parallel in respect to each other and hence create no fringes $[10,11]$. This case is referred to as the 'heterodyne condition'. Alignment of SHS involves the rotation of one or both of the transfer mirrors to set the angles such that a chosen wavelength satisfies the heterodyne condition $\left(\lambda_{0}\right)[10,11]$. At all other wavelengths $\left(\lambda=\lambda_{0}+\Delta \lambda\right)$ there is a dispersive rotation introduced to the merging wavefronts and results in the formation of a 2-D Fizeau fringes with a frequency dependent on $\Delta \lambda$ [10]. The data fringe pattern 

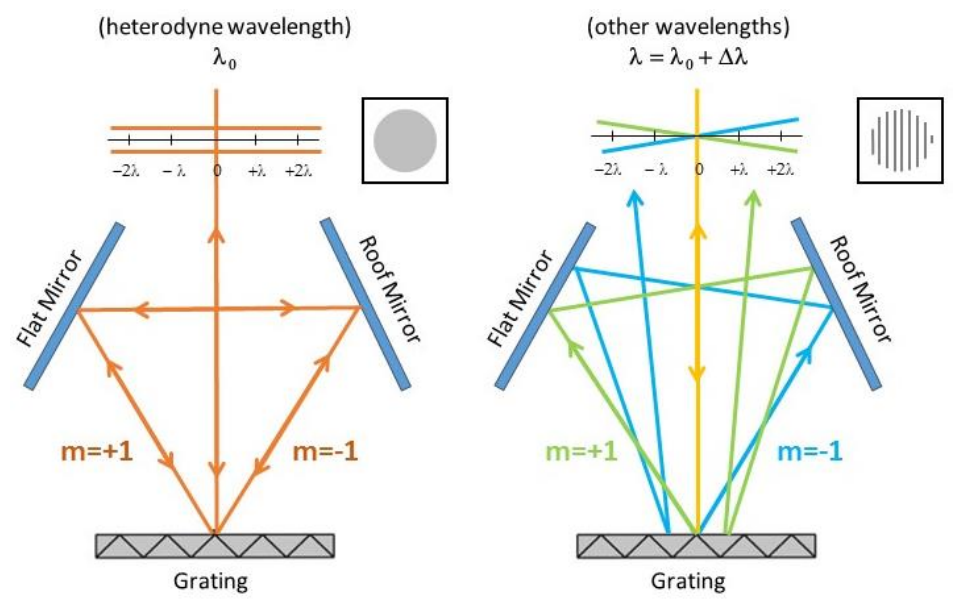

Figure. 2 (Left) PHI is based on a remarkably simple cyclical design. The incoming collimated light hits the grating at normal incidence, splits to two orders, travel in opposite directions, and diffract off the grating before exiting. For the tuned wavelength $\lambda_{0}$ everything is symmetric, there is no path or phase differences between the emerging beams. (Right) However the exiting wave fronts of other wavelengths $\left(\lambda=\lambda_{0}+\Delta \lambda\right)$ are tilted in respect to each other producing a fringe pattern.

forms at a location called the Fringe Localization Plane (FLP) and is imaged onto a 2-D imaging detector [10]. FLP is defined by following the diverging exiting beams from the interferometer and is a virtual plane located at distance $\mathrm{z}_{0}$ behind the grating on the $\mathrm{z}$-axis [5]. In the small angle approximation, the FLP position can be estimated as

$$
z_{0} \approx \frac{L}{2 \cos ^{2} \beta_{0}}
$$

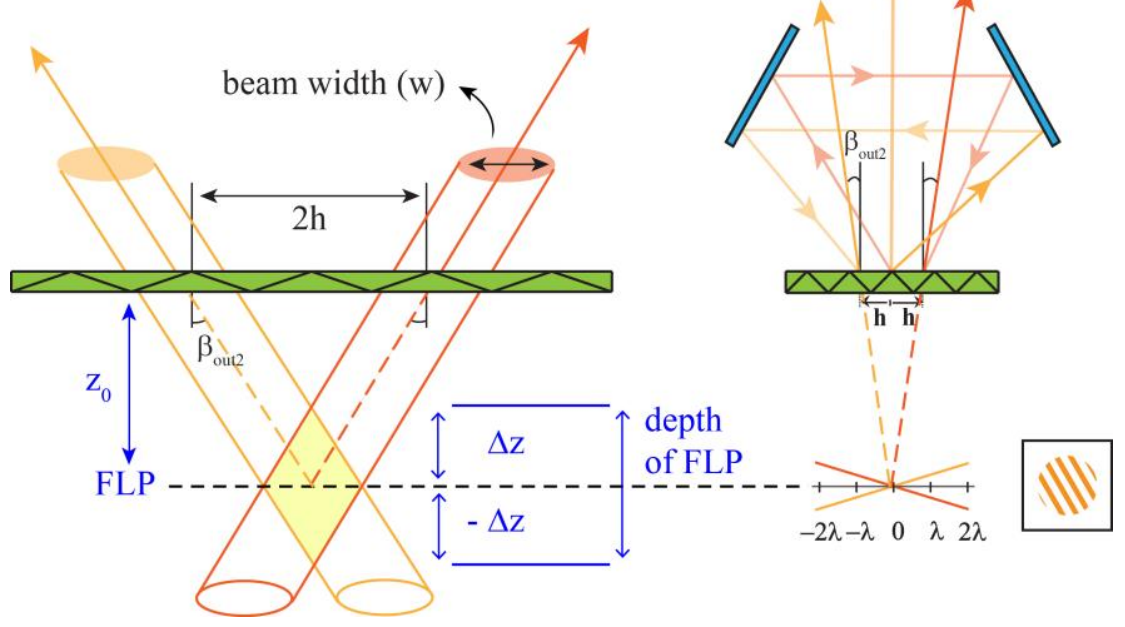

Figure. 3. In reflective SHS, the Fringe Localization Plane (FLP) is the location that the detector presumes the beams are intersecting which is an image of the imaginary FLC located at $\mathrm{z}_{0}$ distance behind the grating. The depth of FLP focus is the distance that detector can image while maintaining an acceptable fringe contrast. 
where $\mathrm{L}$ is the average optical path inside the SHS interferometer. In the small angle approximation the recorded interference pattern on the detector at wavelength $\sigma=1 / \lambda$ from the two $\mathrm{m}= \pm 1$ orders is defined as:

$$
\begin{aligned}
& I\left(\mathrm{x}, \mathrm{y}, \mathrm{z}_{0}, \sigma\right)=\int_{\sigma_{1}}^{\sigma} I(\sigma) d \sigma\left[1+\cos \left(2 \pi\left(f_{x} x+f_{y} y+f_{z} z_{0}\right)\right)\right] \\
& f_{x} \approx-4 \delta \sigma \sin \beta_{0} \\
& f_{y} \approx 2 \sigma \phi \\
& f_{z} \approx 2 \sigma \sqrt{1-4 \sin \beta_{0}^{2}(\delta \sigma / \sigma)^{2}}
\end{aligned}
$$

Where $I(\sigma)$ is the spectral intensity of the input light, $\phi$ is the cross angle between the interferometer mirror and the diffraction plane and-and $\sigma_{2}$ defines the bandpass boundary. The two-dimensional Fourier transform of $I\left(x, y, z_{0}, \sigma\right)$ recovers the power spectrum [5].
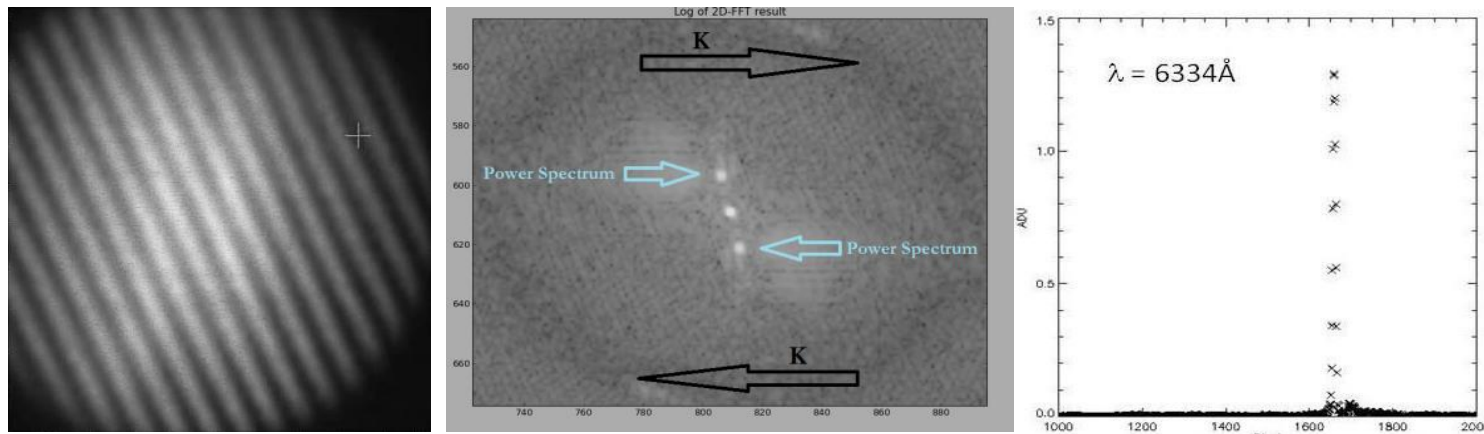

Figure 4. (Left) The interference pattern from the Ne lamp at $6334 \AA$ wavelength taken from Khayyam. The cross tilt would separate the lower wavelengths from the upper wavelengths in the Fourier Transform process in respect to the homodyne wavelength and solves the aliasing problem. The cosmic ray and flat field has been corrected. Flat fields in interferometers are usually obtained by blocking each arm and allowing the flat field image of only one arm reaching to the camera. The final flat field is the average of all separate images taken of all arms. (Middle) The 2D Fourier Transform of the data interference pattern from the Ne lamp at $6334 \AA$ wavelength. The top half and the bottom half represent the same power spectra with different wave vector $(\mathrm{K})$ direction. The circular pattern around the power spectra are the remnants remained from the flat field. The central zero frequency burst and the two identical power spectra are shown in center. (Right) The power spectrum of the Ne lamp at $6334 \AA$ wavelength.

The resolving power (R) and FOV of the SHS, in small angle approximation, are defined by

$$
\begin{aligned}
& R=\frac{\lambda_{0}}{\Delta \lambda}=4 m W_{g} G \\
& F O V \approx \frac{2 \pi}{R} \times \frac{W_{g}}{D_{T}}
\end{aligned}
$$

where $\mathrm{m}$ is the diffraction order, $\mathrm{W}_{\mathrm{g}}$ is the width of the beam on the grating, $\mathrm{G}$ is the grating groove density, and $\mathrm{D}_{\mathrm{T}}$ is the diameter of the collecting area of the input telescope, if one is used [1,7]. SHS can be used either at the focus of a telescope of diameter $\mathrm{D}_{\mathrm{T}}$ or as a stand-alone instrument, in which case $\mathrm{D}_{\mathrm{T}}=\mathrm{W}_{\mathrm{g}}$ in equation (2). If the $\mathrm{f} / \#$ of the telescope and collimation optics is equal, then the FOV is reduced by the ratio of $\mathrm{W}_{\mathrm{g}} / \mathrm{D}_{\mathrm{T}}$ while the collecting area is increased by the same amount. At a given grating density, the final beam width on the grating defines the resolving power following equation (2). The ability to potentially couple Khayyam to two different apertures size telescopes in future and exchanging the gratings can, in principle, provide a wide range of setups achievable in a very short time [5]. 
The SHS bandpass is determined by $\pm \Delta \lambda_{B}$ around $\lambda_{0}$, bandpass and is defined by the highest resolved spatial fringe frequency number on the detector of a given format. The theoretical limit of the bandpass is set by the Nyquist limit established by the number of pixels on the detector used and is given by

$$
\Delta \lambda_{B}=\frac{\lambda_{0}}{\frac{R k}{N}-1}
$$

where $\Delta \lambda_{\mathrm{B}}$ is the half bandpass, $\mathrm{R}$ is the resolving power, $\mathrm{k}$ is the minimum number of pixels required to resolve one fringe (for the Nyquist limit: $\mathrm{k}=2$ ), and $\mathrm{N}$ is the total number of pixels along the detector [1]. The practical bandpass limit is less than this due to a variety of factors such as the phasing of pixels and fringes, or focus. The short spectral response has made SHS instruments useful primarily for studies of single emission line features or molecular bands. Through various methods, SHS can be tuned in bandpass to extend the usable wavelength range over a much broader spectral range $[1,6]$.

\section{KHAYYAM: FIELD TSHS AT MT. HAMILTON}

Over the past two decades, a series of SHS instruments have been constructed and successfully operated in either the Michelson design [3, 4] or reflective design [1, 5]. In 2009, we started developing Khayyam to be able to observe inside the UC Davis campus, by coupling the instrument to an off-axis heliostats. The heliostat on top of the roof of the UCD laboratory at second floor was designed to use two computer controlled rotating flat mirrors, to guide the light into the lab on the optical table where Khayyam was setup [7]. With the extended delivery delay in the UC Davis heliostat system, in late 2010, Khayyam was transferred to Mt. Hamilton. The main targets that Khayyam is aimed to observe are comets and planetary atomosphers $[1,3]$. The combination of low velocity, angularly extension, low surface brightness, low temperatures, and complex and spatially variable composition at these targets can be challenging for grating spectroscopic observers but then well suited to SHS capabilities. Moreover most of these targets are highly temporally variable on scales from hours to months, which necessitate a high temporal observation. This is possible with SHS instruments coupled to small aperture telescopes that are not in high demand in comparison to large aperture telescopes such as Kick. Over the next four-years (2010-2015), a continuous progress was made toward development of a field tunable reflective SHS coupled to fixed focal plane shared by the $0.6 \mathrm{~m}$ Coudé Auxiliary Telescope (CAT) and the $3 \mathrm{~m}$ Shane telescope.

Khayyam has been installed and located in the old Laser Pool at a fixed location $\sim 3 \mathrm{~m}$ below the shared Shane/CAT coudé focus. Shane is the largest aperture telescope on Mt. Hamilton and it shares a common focal plane with CAT at coudé configuration (figure 5). CAT is a $0.6 \mathrm{~m}$ Cassegrain telescope rigidly mounted to the inside of the dome of the $3 \mathrm{~m}$ Shane telescope that is fed by an external siderostat located next to the dome (figure 5 and 6). The two $0.6 \mathrm{~m}$ CAT and $3 \mathrm{~m}$ Shane
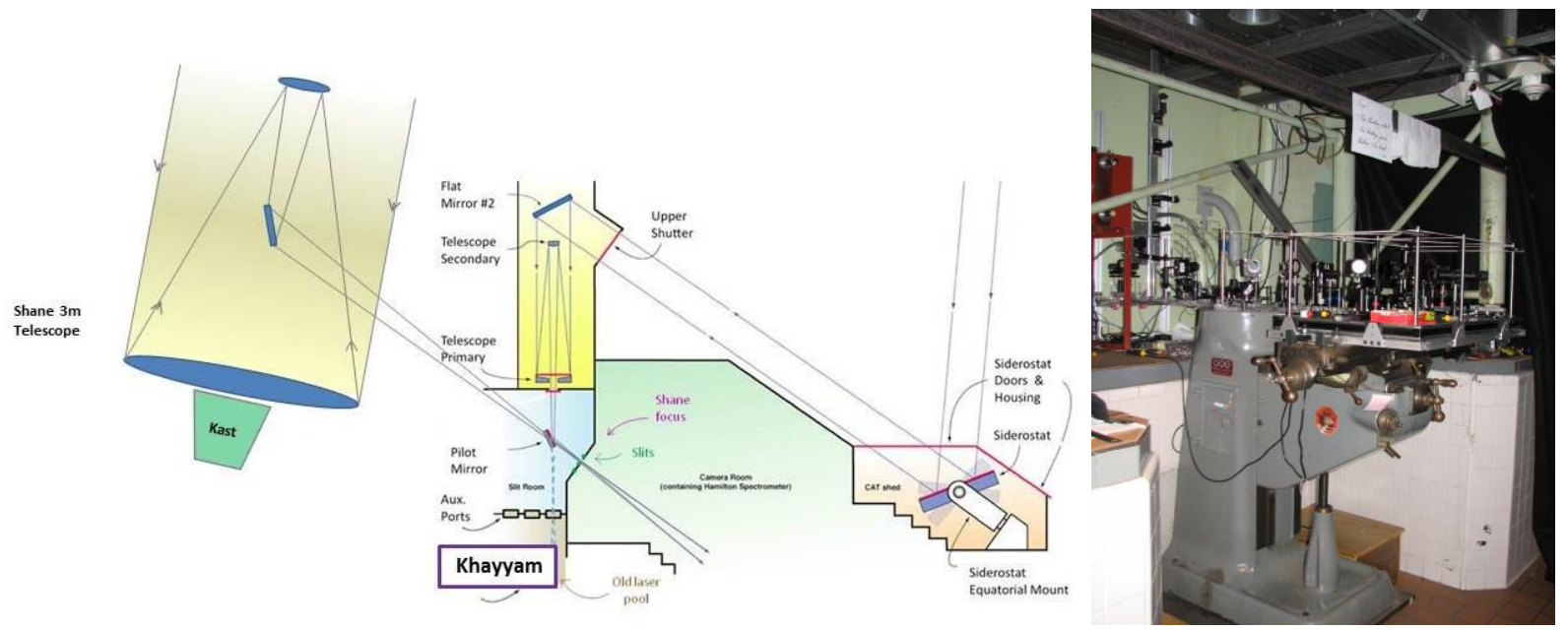

Figure 5. (left) Khayyam is located below the shared focal plane of the .6m CAT and the 3m Shane Telescope. This unique location would enable Khayyam to potentially use both telescopes in future. (right) Khayyam is currently coupled only to the $0.6 \mathrm{CAT}$ and is setup on the lower half of a Bridgeport milling machine located on a concrete slab which provides stability and complete 3 -axis mobility and limits the vibrations in the system. 
telescopes at coudé configuration share a focal plane that provides an opportunity for using the same instrument with two telescopes [6,7]. The instrument pier consists of the lower half of a Bridgeport milling machine which provides us with complete 3-axis mobility in the instrument placement (figure 5 and 6). The milling table is located on a concrete slab that provides stability and limits vibrations in the system.
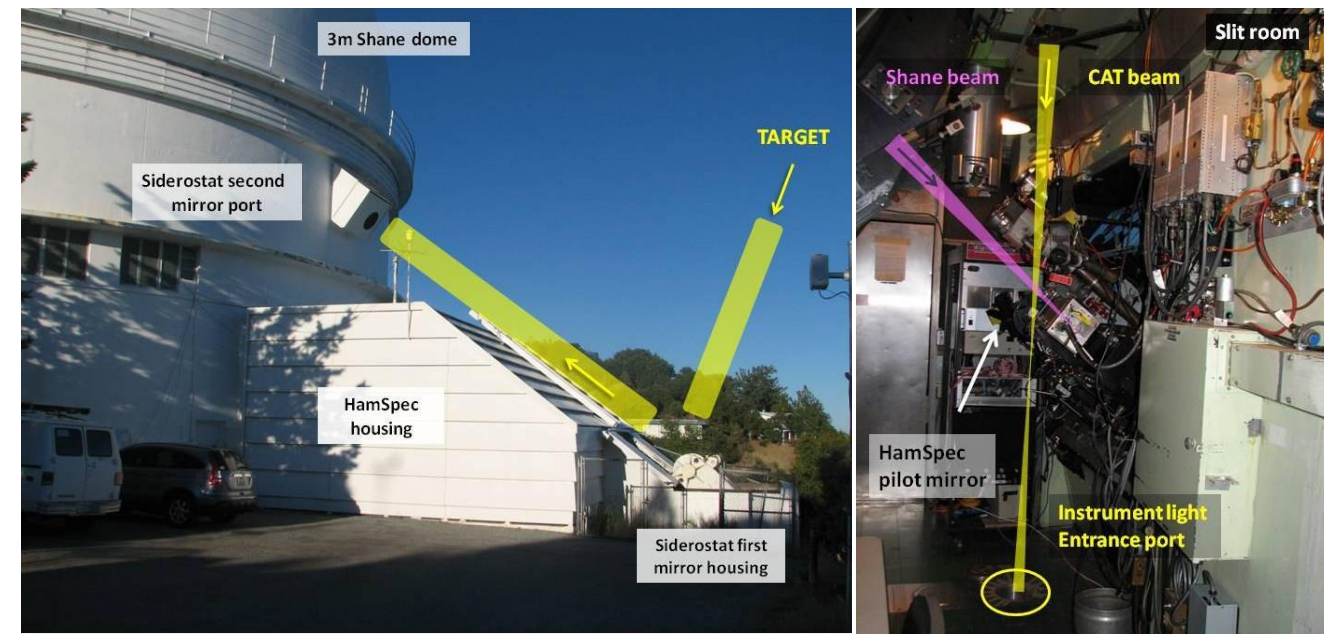

Figure 6. (left) The optical layout of the beam into the CAT. (right) The optical layout of the beam from the CAT and the Shane into the slit room and into the instrument.

\subsection{Alignment and guide camera configuration}

Khayyam can be coupled to both telescopes by using the shared focus of CAT and Shane $[8,9,12]$. The f/36 CAT beam comes to a focus in a 'slit room' where it can be directed by a pilot mirror into the slit-plate of the Hamilton Spectrograph (HamSpec), a traditional cross-dispersed Echelle spectrometer housed in a large camera room off to one side of the beam (figure 6). Shane at coudé configuration will bring the light into the slit room to HamSpec with the same f/36 as CAT. $\mathrm{CAT}$ and Shane at coudé configuration offer identical étendue \& resolving power options over different fields of view and the common f\# enables their interchange without affecting the downstream optics of Khayaym [5].

For safety, stability, and logistical reasons we decided to couple Khayyam only to CAT by transferring the CAT focus into the Laser Pool on Khayyam's FOV stop on a rail mounted at the Bridgeport base [12]. The HamSpec pilot mirror was removed to allow the beam to pass through the floor of the slit room and into the Laser Pool into Khayyam. Incoming light from the CAT follows a vertical optical rail and is folded by a right angle mirror onto a reimaging system that focuses the beam onto a FOV stop that matches the f/\# input acceptance angle of the SHS. The facility guide camera is mounted in the slit room near to the HamSpec pilot mirror and images the focal plane of the telescope on the entrance slits and is only usable if the beam incident on the HamSpec slit-plate. For this reason we has to design our own independent guiding system that combines the need for rejecting out of band emission with the need for on-axis guider. The off-axis guide camera is fed by the reflected beam off a broadband dichroic hot-mirror prior to the FOV stop. A small folding mirror guides the reflected light from the filter into a wide-field guide camera that images the target within a $9^{\prime} \times 7^{\prime}$ FOV. The output of the camera is displayed on the instrument PC in real time and it is used to manually control the CAT guiding system [12]. The combination of the grating groove density and the final beam width on the grating defines the resolving power [5]. For our initial setup the beam width was set to $1 \mathrm{~cm}$, which provides sufficient bandpass to cover molecular bands at resolving power high enough to separate overlapping spectral features. This width also allows 1:1 magnification of the FLP onto the CCD. Once the beam strikes the 1200 (grooves $/ \mathrm{mm}$ ) grating, two diffracted orders $( \pm 1)$ circulate antisymmetrically through the mirrors and return to the grating for second time at a location below the first incident beam. A final folding mirror sends the light to the FLP imaging lens where the FLP is imaged on the CCD camera [5]. For the tuning purposes, the mirrors are installed on rotation stages [5, 8].In order to make the entire instrument an all-reflective system, the imaging system would be replaced by an all reflective offner-system [11]. A detailed explanation of how SHS instruments are aligned and work can be found in $[5,7]$. 

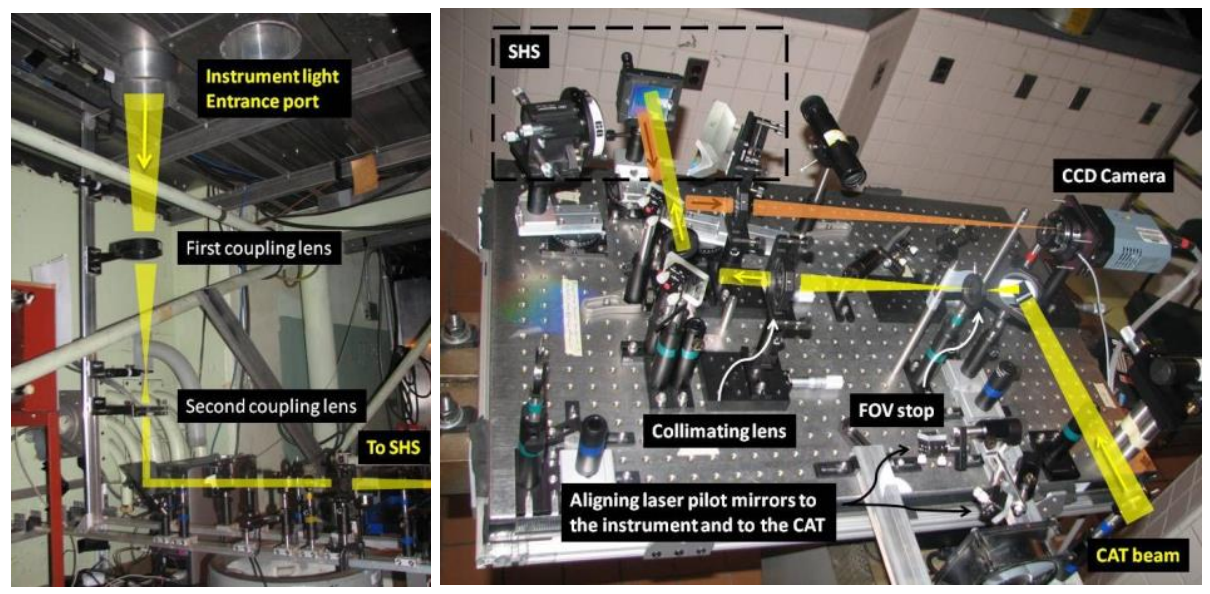

Figure 7. (left) Coupling system optical layout. Science beam splits by the narrow band filter into two beams, one trough the guide camera and one trough the instrument. The calibration lamp assembly is flipped out of the optical axis in this picture. (right) All the optics outside the TSHS market box are imaging optics which indicates the small size of the instrument.

\subsection{Data reduction procedure}

The data optioned from SHS by a CCD camera are a set of Fizeau fringes in result of wavefront rotation occur in the SHS interferometer. These fringes represent the accumulation of an optical path difference between the crossed wavefronts for any non-heterodyne wavelength. In Khayyam these interference fringe images are saved in the Flexible Image Transport System (FITS) format which is a standard astronomy format used to transfer images and catalogs in an information-rich format $[5,13]$. At every given setup, the light outside the SHS bandpass would still reach the detector, and because it cannot be resolved it contributes noise in the Fourier transform of the interference pattern. One way around this is to use narrow bandpass filers in front of the entrance aperture or the imaging camera to filter the light out of the bandpass. If the narrow bandpass filter is used with a white light source, it would result to a barber-pole alike pattern due to the constructive interference of all wavelengths at the vicinity of the zero Optical Path Durance (OPD). The fringes for all the wavelengths within the bandpass of the narrowband filter exists everywhere but as we go farther from zero OPD the difference between the fringe frequencies would result in washout, diminishing the contrast until we have no detectable fringe pattern [5]. In order to obtain the power spectra from the interference fringes and ultimately calibrate our data, we have developed a data reduction program in Python. Figure 8 shows the diagram of basic processes that are used to reduce the power spectra from the recorded SHS interference data.
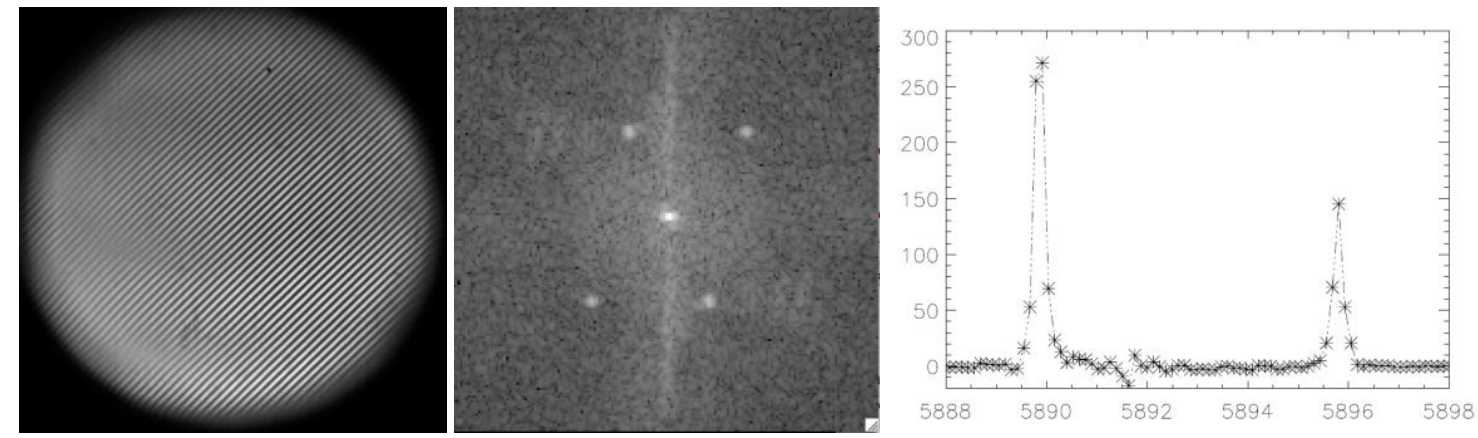

Figure 7. (left) Fringe pattern from Na Hollow Cathode Lamp when Khayyam is tuned to the vicinity of the Na D lines at $\lambda_{\mathrm{D} 1}=5895.9 \AA, \lambda_{\mathrm{D} 2}=5889.9 \AA$. (middle) The reduced power spectra shows both lines indicating both fringe pattern are present in the data. The top half of the 2D FFT is the repetition of the bottom half which an inverse wavevector sign. (right) The Na D lines' power spectra: the separation of $\Delta \lambda=5.97 \AA$, indicates a resolving power of $\mathrm{R} \sim 48000$ which is same with the calculated $\mathrm{R}$. 


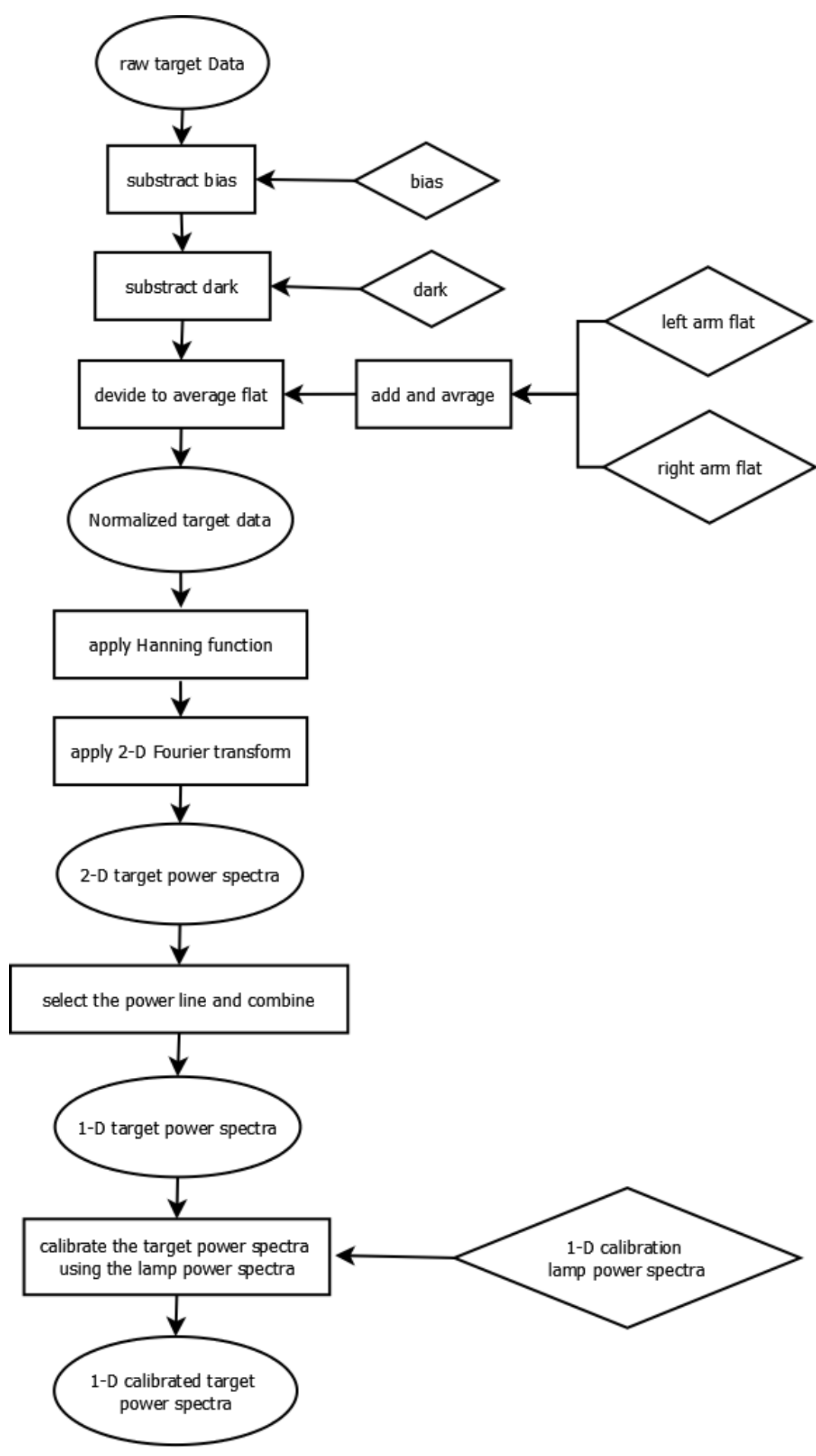

Figure 8. In the data reduction pipeline, the target data is normalized using the bias, dark and flat data taken prior or after the target data. The flat is taken separately from each arm and the average flat is used to normalize the target data. Hanning function is applied before the 2 dimensional Fourier transform generates the power 
Table 1. The core of Khayyam is a tunable reflective SHS. It is coupled to the 0.6m CAT in Mt. Hamilton and offers integrated spectra at high spectral resolving power (R 50,000), wide field of view (FOV $40 \operatorname{arcsec})$ and small bandpass $\left(\Delta \lambda_{\mathrm{B}} \sim 40 \AA\right.$ ) .

\begin{tabular}{|c|c|}
\hline Technique & $\begin{array}{l}\text { The core of Khayyam is a tunable reflective SHS, a cyclical interferometer based on } \\
\text { wave-front phase differences. }\end{array}$ \\
\hline Configuration & $\begin{array}{l}\text { Khayyam offers integrated spectra at high spectral resolving power }(\mathrm{R} \sim 50,000) \text {, wide field } \\
\text { of view (FOV } 40 \text { arcsec) and small bandpass }\left(\Delta \lambda_{\mathrm{B}} \sim 40 \AA\right) \text {. }\end{array}$ \\
\hline Coupling telescope & $\begin{array}{l}\text { The Coude Auxiliary Telescope (CAT): a } 0.6 \mathrm{~m} \text { Cassegrain telescope rigidly mounted to } \\
\text { the inside of the dome of the } 3 \mathrm{~m} \text { Shane telescope that is fed by an external heliostat-like } \\
\text { system located next to the dome }\end{array}$ \\
\hline CAT Plate Scale & $9.45(\operatorname{arcsec} / \mathrm{mm})$ \\
\hline Aligning laser & $\begin{array}{l}\text { Low power diode laser } \sim 5 \mathrm{~mW} \\
\text { Melles griot } 1.00-1.60 \mathrm{~mW} 632.8 \mathrm{~nm} \text { (red) Heilum Neon Laser, Model: } 05-L H R-911\end{array}$ \\
\hline Input beam f/\# & $\begin{array}{l}\text { f/36 on the shared focus } \\
\text { f/ } 50.6 \text { on the Khayyam's FOV stop }\end{array}$ \\
\hline Plate Scale on the FOV Stop & $6.7(\operatorname{arcsec} / \mathrm{mm})$ \\
\hline opening size of FOV aperture & $5.73(\mathrm{~mm})$ \\
\hline Collimating lens & $\begin{array}{l}\text { Thorlabs UV fused Silica Plano-Convex lens, uncoated } \\
\text { LA4782 - } \mathrm{f}=500(\mathrm{~mm}), 2 \text { inch diameter }\end{array}$ \\
\hline Grating & $\begin{array}{l}\text { Customized Non-Blazed symmetric reflective grating from Horiba Jobin Yvon } \\
\mathrm{G}=1200 \text { groves } / \mathrm{mm} \\
\text { Model: } 520-24-330 \\
\text { Spectral Range: } 400-1300\end{array}$ \\
\hline Beam width on the grating & $10 \mathrm{~mm}$ \\
\hline Resolving power (l/dl) & 49000 \\
\hline Field of View (FOV) of SHS & $39 \operatorname{arcmin}, 19 \operatorname{arcsec}$ \\
\hline FOV on sky & $39 \operatorname{arcsec}$ \\
\hline $\operatorname{Bandpass}\left(\Delta \lambda_{\mathrm{B}}\right)$ & $38.11 \AA$ \\
\hline $\begin{array}{l}\text { zo, Fringe Localization Plane } \\
\text { (FLP) location }\end{array}$ & $\begin{array}{l}\text { The diverging beams exit the interferometer. FLP is located at } \mathrm{z}_{0} \text { behind the grating. } \\
\mathrm{z}_{0}=14.8 \mathrm{~cm} \text { behind the grating }\end{array}$ \\
\hline FLP imaging lens & $\begin{array}{l}\text { Newport Corporation } \\
\text { Plano-Convex Lens, N-BK7, } 50.8 \mathrm{~mm} \text { Diameter, } 250 \mathrm{~mm} \text { EFL, 430-700 nm } \\
\text { MODEL: KPX202AR.14 }\end{array}$ \\
\hline
\end{tabular}

\begin{tabular}{|l|l|}
\hline Filters & $632.80, \mathrm{FWHM}=3 \pm 0.5$ \\
& $634.70, \mathrm{FWHM}=11.2$ \\
& $630.00, \mathrm{FWHM}=1.0$ \\
& $590.30, \mathrm{FWHM}=9.60$ \\
& $589.08, \mathrm{FWHM}=0.2$ \\
& $589.70, \mathrm{FWHM}=0.47$ \\
& $590.06, \mathrm{FWHM}=0.52$ \\
& $557.70, \mathrm{FWHM}=1.0$
\end{tabular}




\begin{tabular}{|c|c|}
\hline Guide camera FOV & $9 \times 7 \operatorname{arcmin}$ \\
\hline $\begin{array}{l}\text { beam splitter for guide camera } \\
\text { beam vs. interferometer }\end{array}$ & Thorlabs, FM201 - Wide Band Hot Mirror, Diameter = 2", $1 \mathrm{~mm}$ Thick \\
\hline Calibration lamp & $\begin{array}{l}\text { UVP penray lamps: } \\
\text { Argon model 90-0013-01 } \\
\text { Krypton model 90-0014-01 } \\
\text { Neon model 90-0015-01 } \\
\text { Neon-Mercury model 90-0016-01 } \\
\text { Xenon model 90-0017-01 } \\
\text { Hg model 90-0079-01 } \\
\text { Zince Axial leads, 8" (203mm) model 90-0069-06 } \\
\text { Cadmium Axial leads, 8" (203mm) model 90-0071-06 }\end{array}$ \\
\hline Mirror rotation stages & $\begin{array}{l}\text { Newport, Model: } 481-\mathrm{A} \\
\text { Rotation Stage, } 360^{\circ} \text { Coarse, } 5^{\circ} \text { Fine, Micrometer }\end{array}$ \\
\hline
\end{tabular}

Table 2. By changing the telescope aperture, beam width on the grating or the grating density, Khayyam's setup can be changed to match varies FOV and R over a given tune. At the moment Khayyam is only coupled to CAT but we also have included the numbers if it was also coupled to Shane. The numbers provided in the table are for when the instrument is tuned to the HeNe laser wavelength at $\lambda_{0}=6328$ using a 1220 element CCD detector.

\begin{tabular}{|c|c|c|c|c|c|c|}
\hline $\mathbf{W}(\mathbf{m m})$ & $\begin{array}{c}\mathbf{G} \\
(\text { grooves/mm) }\end{array}$ & $\mathbf{R}$ & $\begin{array}{c}\text { Total bandpass } \\
(\AA)\end{array}$ & $\begin{array}{c}\text { SHS input } \\
\text { acceptance angle }\end{array}$ & $\begin{array}{c}\text { SHS-CAT } \\
\text { FOV }\end{array}$ & $\begin{array}{c}\text { SHS-Shane } \\
\text { FOV }\end{array}$ \\
\hline 5 & 1200 & 24000 & 275.8 & $55^{\prime} 37^{\prime \prime}$ & $27^{\prime \prime}$ & $5 "$ \\
\hline 10 & 1200 & 48000 & 136.4 & $39^{\prime} 19^{\prime \prime}$ & $39^{\prime \prime}$ & $7^{\prime \prime}$ \\
\hline 20 & 1200 & 96000 & 67.8 & $27^{\prime} 48^{\prime \prime}$ & $55^{\prime \prime}$ & $11^{\prime \prime}$ \\
\hline 5 & 600 & 12000 & 564.06 & $1^{\circ} 18^{\prime} 39^{\prime \prime}$ & $39^{\prime \prime}$ & $7 "$ \\
\hline 10 & 600 & 24000 & 275.8 & $55^{\prime} 37^{\prime \prime}$ & $55^{\prime \prime}$ & $11^{\prime \prime}$ \\
\hline 20 & 600 & 48000 & 136.4 & $39^{\prime} 19^{\prime \prime}$ & $1^{\prime} 18^{\prime \prime}$ & $15^{\prime \prime}$ \\
\hline
\end{tabular}

Table 3. SHS instrument can provide an alternative option suitable for small aperture telescopes to provide high spectral R capability at high FOV, étendue, and grasp. By comparing SHS to a selection of commonly used high-R spectrometers in optical and UV wavelengths, it can be seen that SHS can utilize small aperture telescope to achieve comparable high R spectra at wide FOV. This ability puts SHS among the highest étendue instruments and in supreme group for grasp values. These conditions in addition to small physical size of SHS make them candidates favorable for space missions.

\begin{tabular}{|c|c|c|c|c|c|c|}
\hline Telescope & $\begin{array}{c}\text { Diameter } \\
\text { (m) }\end{array}$ & Instrument & $\begin{array}{c}\text { FOV } \\
\left(L^{\prime} W^{6}\right)\end{array}$ & $\begin{array}{l}\text { Resolving } \\
\text { Power }\end{array}$ & $\begin{array}{c}\text { Étendue } \\
\left(\mathrm{m}^{2} \operatorname{arcsec}^{2}\right)\end{array}$ & $\begin{array}{l}\text { Grasp } \\
\times 10^{5}\end{array}$ \\
\hline Siderostat & 0.015 & Khayyam & $1927 \times 1927$ & 72000 & 655 & 470 \\
\hline CAT & 0.6 & Khayyam & $48 \times 48$ & 72000 & 655 & 470 \\
\hline Shane & 3.0 & Khayyam & $9.5 \times 9.5$ & 72000 & 655 & 470 \\
\hline Keck & 10 & HIRES & $8 \times 0.7$ & 47000 & 440 & 206 \\
\hline $\begin{array}{l}\text { McDonald } \\
\text { Observatory }\end{array}$ & 2.7 & $\begin{array}{c}\text { Coudé } \\
\text { Spectrograph }\end{array}$ & $8 \times 1.2$ & 60000 & 55 & 33 \\
\hline $\begin{array}{l}\text { Apache Point } \\
\text { Observatory }\end{array}$ & 3.5 & ARCES & $1.3 \times 0.8$ & 27000 & 10 & 2.7 \\
\hline Kueyen & 8 & UVES & $0.45 \times 8$ & 80000 & 180 & 144 \\
\hline Hubble & 2.4 & STIS & $0.5 \times 52$ & 9000 & 117 & 10 \\
\hline Rosetta & 0.04 & ALICE & $180 \times 7200$ & 137 & 1630 & 2.2 \\
\hline
\end{tabular}




\subsection{Tuning in wavelength}

The tuning range of Khayyam is limited in three ways: 1) the size of the mirrors relative to the changing dispersion angle from the grating, 2) the blaze function of the grating, and 3) the depth of field at the FLP, which moves in response to changes in the internal angles, which affect the path length. The bandpass change in Khayyam is accomplished through

$D 1=5895.92 \AA$
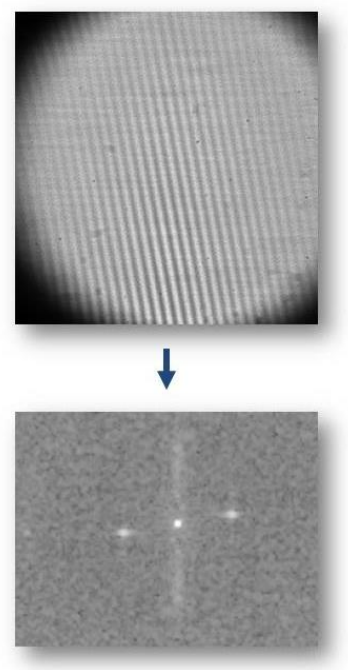
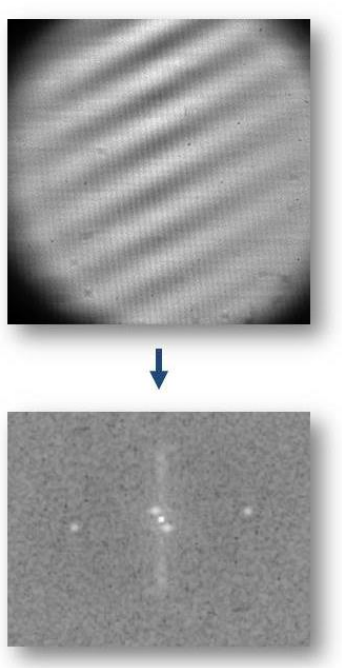

$\mathrm{D} 2=5889.95 \AA$

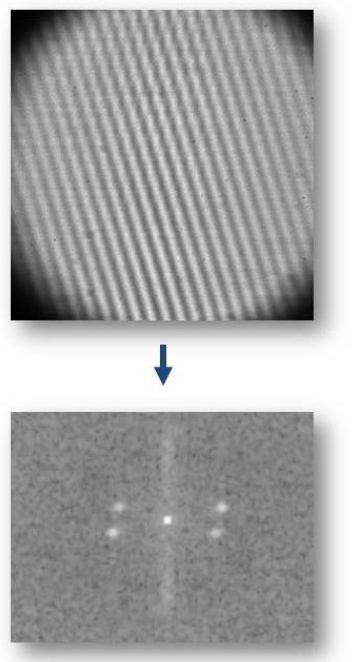

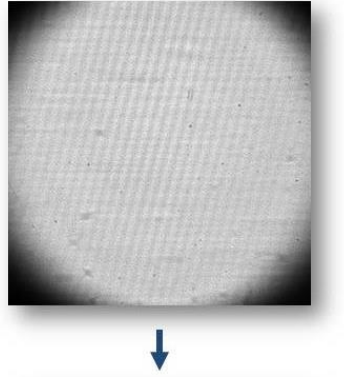

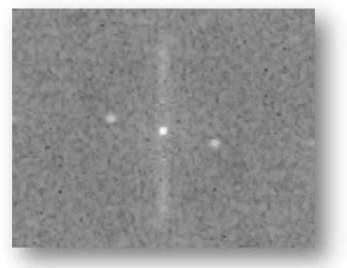

Figure 9. The fringe pattern sequence when tuning from Na lamp D1 line: $\lambda_{\mathrm{D} 1}=5895.9 \AA$, to the direction of $\lambda_{\mathrm{D} 2}=$ $5889.9 \AA$. As seen in the reduced power spectra under the fringe patterns, the horizontal fringe frequency for each emission line indicates the horizontal location of the power spectra and the vertical tilt of the fringes indicate the vertical separation in respect to the center of the $2 \mathrm{D}$ FFT.

rotating the mirrors, using controlled rotation stages to change the heterodyne wavelength to a different wavelength $[5,7$, 8]. We adopted the strategy of targeting a smaller bandpass for a single configuration, with expansion coming from rotating mirrors, changing the groove density of the grating, the width of the collimated beam, and the focal length of the input and output optics. Every tune modification requires the symmetric rotation of both mirrors in order to maintain the same position of the interference pattern at the FLP and on the detector. Hence, any inaccuracy in the symmetry of the rotation will move the image slightly, with the offset accumulating as the number of tuning motions is increased. An additional factor comes from the precision of the alignment of the center axis of the rotation stages to the reflecting faces of the mirrors. Cross tilt in the flat mirror (for de-aliasing) and vertical shifts of the beams (from cross tilt or internal mechanical shifts in the input optics) in the roof mirror can introduce the same effect. Figure below shows the fringes when tuning in wavelength over the narrow range between two $\mathrm{Na} D$ lines. As shown, the fringe frequency for every emission line depends on where is the tune or the heterodyne wavelength $\left(\lambda_{0}\right)$.

\subsection{The challenge of the spider-pattern shadow over the interference fringe pattern}

Both CAT and Shane telescopes are Cassegrain Telescopes and contain supporting spider pattern for their secondary mirrors in collimated light. Since the CCD camera of Khayyam is focused on the fringe localization plane (FLP) and not on the primary mirror, the telescope spider pattern presents a shadow on the data fringes [5,9]. The spider pattern shadow goes through the interferometer and the diffracted shadow of the spider pattern is present on the FLP and is imaged on the CCD. The spider pattern is an overlap shadow on the fringe pattern at any present wavelength. This complicates the data reduction process and the calibration process. To investigate the effect of the spider pattern in the output power spectrum, we have installed calibration lamps outside the CAT dome to look at semi monochromatic collimated light on Khayyam that contains the spider pattern. Figure 10 shows the calibration lamp setup on the edge of the $3 \mathrm{~m}$ Shane telescope. Even though the lamp light is not perfectly collimated, it clearly shows the shadow on Ne line. Since he spider shadow goes through the interferometer, just like the beam itself, it will be present for every wavelength that is present in the beam diffracted along the diffraction plane on the FLP. The Clean Method from Radio-astronomy can be used to go back and forward with the FFT process to try to eliminate the shadow at every wavelength. Using the Clean Method for visible 
wavelength in this case results in a reduction that is far more complex and the power extraction appears to be more compromised in spectral domain than for isolated emission lines. For future development two other options for mitigating the spider can be explored 1) defocusing the telescope at the SHS entrance aperture and 2) inserting a cylindrical lens into the collimated beam to focus the axis perpendicular to the sky at the FLP, thereby destroying the coherent image of the spider. The latter option has the side benefit of providing limited spatial information inside the interferogram.
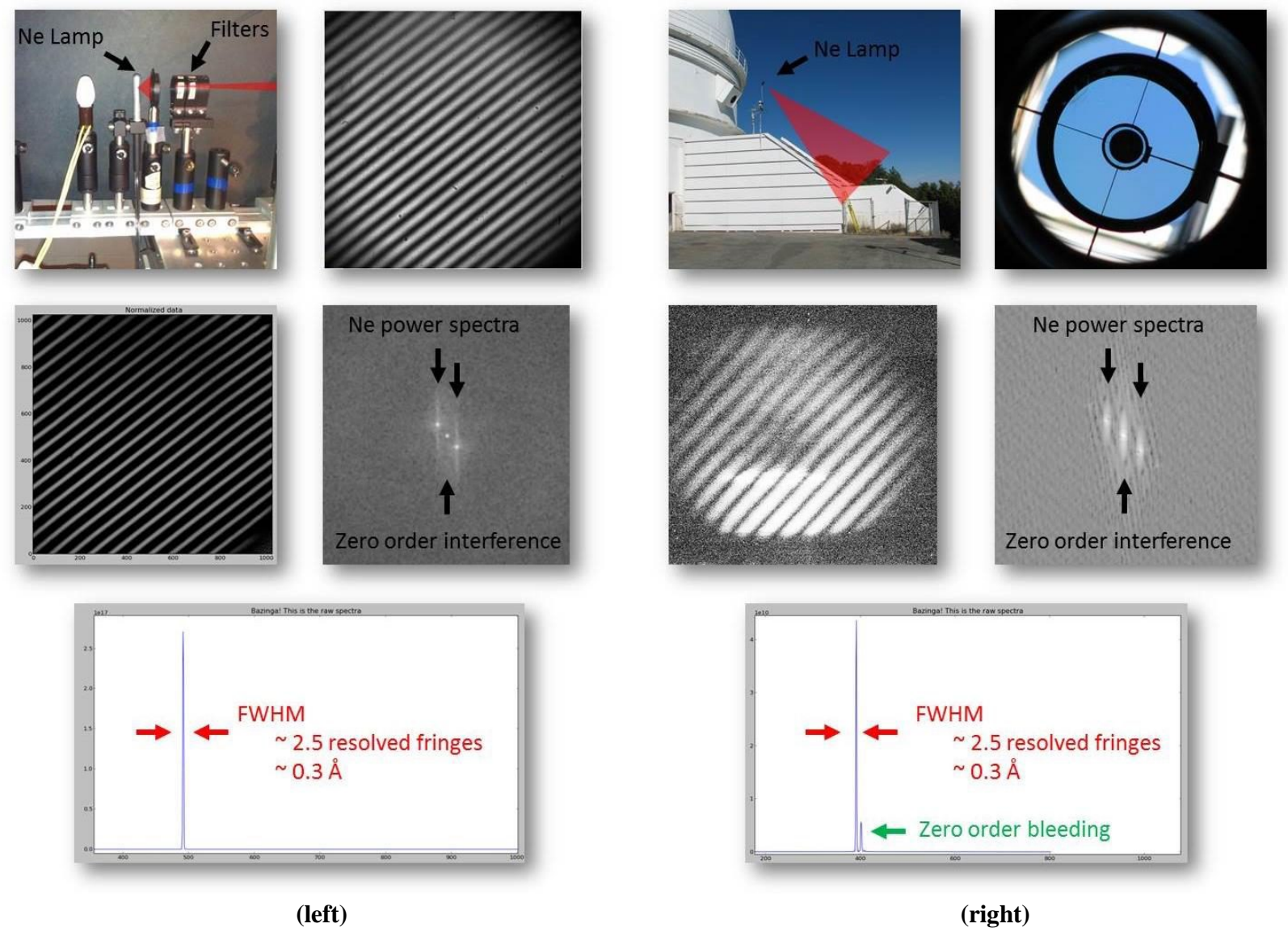

Figure 10. (left) Ne pen-ray lamp installed in the instrument, after the CAT telescope and prior to the interferometer FOV stop. The fringes are from $\mathrm{Ne} 6334 \AA$ and as shown there is no signature of the spider pattern. The reduced spectra shows one emission line and the zero order bleeding is minimum. (right) The Ne pen-ray lamp has been installed on the side of the Shane dome allowing the light to go through CAT and then Khayyam. The fringes are from the same $\mathrm{Ne} 6334 \AA$ at the same tune as the left case. The signature of the spider pattern can be seen in middle of the fringes however because the light is not collimated the spider pattern shadow is not sharp and contains some fringes within it. The reduced spectra shows one emission line and contains the zero order bleeding on top of the Fourier Transform complications due to the spider pattern spikes.

\section{SUMMARY}

The core of Khayyam is a tunable all-reflective spatial heterodyne spectrometer (SHS) and has up to 40 arcsec input field of view, resolving power up to 50000 , and a tunable bandpass covering $400 \mathrm{~nm}>\Delta \lambda_{B}>700 \mathrm{~nm}$. In late 2010, Khayyam was transferred to Mt. Hamilton, a University of California observatory facility located in East of San Jose, CA, to study spatially extended astronomical targets where high resolving power is necessary to separate faint spectral signals, crowded bands, and to sample low $(<10 \mathrm{~km} / \mathrm{s})$ velocities at rapid temporal cadence. From 2010 to 2015 a continuous progress was made toward development of a tunable reflective SHS instrument-telescope configuration to combine all of the capabilities 
necessary to obtain high resolving power visible band spectra of diffuse targets from small aperture on-axis telescopes where significant observing time can be obtained. The majority of the effort was put into coupling Khayyam, to the $0.6 \mathrm{~m}$ Coudé Auxiliary Telescope (CAT) and eliminating the environmental effects such as vibrations. CAT contain supporting spider pattern for its secondary mirror in collimated light and the preliminary data of Khayyam show the shadow of the spider pattern appear in the fringe localization plane (FLP). Multiple data is taken by Khayyam over the course of four year from Venus, Jupiter, Moon, Comet-C2013R1-Lovejoy, day and night sky, San Jose city light but due to the spider shadow unambiguous scientific results was not feasible.

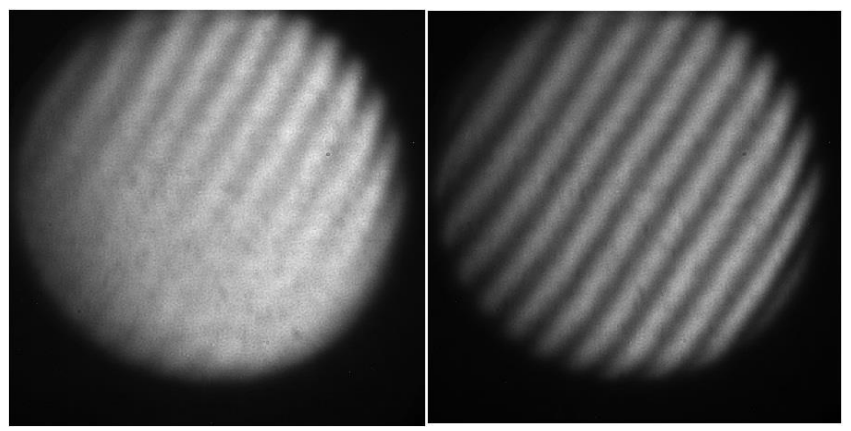

Figure 11. The contrast of the interference fringes would decrease because of high levels of vibration or temperature variation. These two images are Ne lamp data at 2500 seconds exposure time (left) taken with the original coupling system to the telescope (right) taken with the current coupling system to the telescope. The data contrast clearly shows the improvement in the instrument performance.

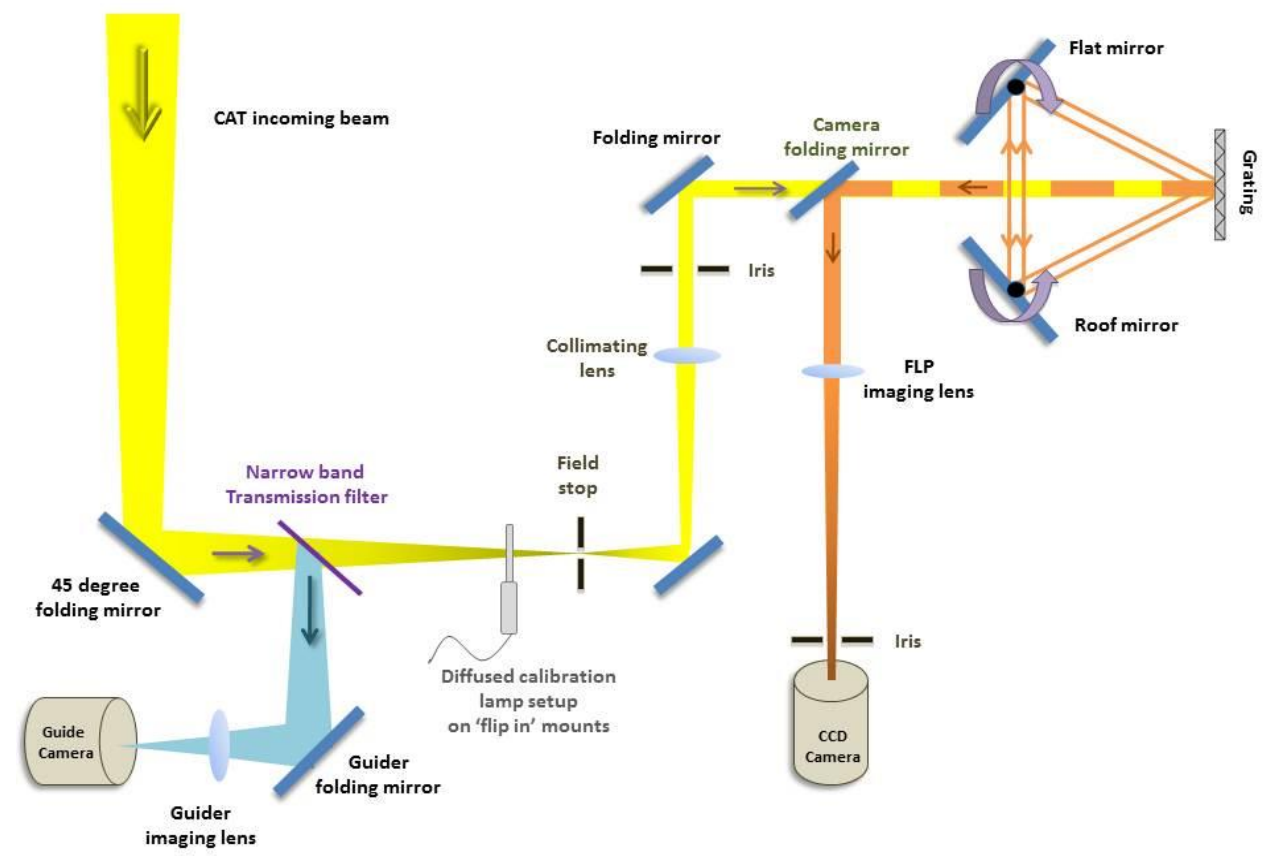

Figure 12. The optical setup of Khayyam imposes upon CAT focal plane at the FOV stop and the off axis guide camera makes use of the out-of-bandpass light. The specifications of the setup is given in Table 1. 


\section{ACKNOWLEDGMENT}

The research was carried out at University of California Davis and the manuscript preparation was carried out at the Jet Propulsion Laboratory, California Institute of Technology, under a contract with the National Aeronautics and Space Administration. This research was supported by NASA grant NNX07AU10G to the University of California, Davis. We gratefully acknowledge the assistance provided by Guillaume Gronoff, Tom Slanger, Kostas Kalogerakis, and Mt. Hamilton staff and business office. Copyright is transferred to SPIE.

\section{REFERENCES}

[1] R. G. Bingham, "Grating spectrometers and spectrographs re-examined," Royal Astronomical Society, Quarterly Journal,, 20, (1979).

[2] R. S. Booth, J. W. Brault, A. Labeyrie et al., "High resolution in astronomy : fifteenth advanced course of the Swiss Society of Astronomy and Astrophysics (member society of the Swiss National Academy of Science)." $217 \mathrm{p}$.

[3] J. M. Hollas, [Modern spectroscopy] Wiley, Chichester West Sussex ; New York(1987).

[4] J. Harlander, Reynolds, R.J., Roesler, F. L., "Spatial heterodyne spectroscopy for the exploration of diffuse interstellar emission lines at far-ultraviolet wavelengths," Astrophysical Journal(396), 730-740 (1992).

[5] S. Hosseini, [Tunable Reflective Spatial Heterodyne Spectrometer: A Technique for High Resolving Power, Wide Field of View Observation of Diffuse Emission Line Sources ] University of California Davis, (2015).

[6] C. R. Englert, D. D. Babcock, and J. M. Harlander, "Spatial heterodyne spectroscopy for long-wave infrared: first measurements of broadband spectra," Optical Engineering, 48(10), 105602-105602-9 (2009).

[7] S. S. Hosseini, A. Gong, D. Ruth et al., "Tunable spatial heterodyne spectroscopy (TSHS): a new technique for broadband visible interferometry." 7734, 77343J.

[8] S. Hosseini, Harris, Walter, "Khayyam: a second generation tunable spatial heterodyne spectrometer for broadband observation of diffuse emission line targets," Proc. SPIE, 8146(814617), 12 (2011).

[9] S. Hosseini, Harris, Walter "First calibration and visible wavelength observations of Khayyam, a tunable spatial heterodyne spectroscopy (SHS)," Proc. SPIE 9147, Ground-based and Airborne Instrumentation for Astronomy, 91478L, 9 (2014).

[10] S. S. Hosseini, [Tunable Reflective Spatial Heterodyne Spectrometer: A Technique for High Resolving Power, Wide Field Of View Observation Of Diffuse Emission Line Sources] University of California Davis, (2015).

[11] S. Hosseini, Gong, Aaron, Ruth, Dustin, Baldis, Hector A., Harris, Walter, "Tunable spatial heterodyne spectroscopy (TSHS): a new technique for broadband visible interferometry,” Proc. SPIE, 7734(77343J), 12 (2010).

[12] S. Hosseini, Harris, Walter, Corliss, Jason, "Khayyam: a tunable spatial heterodyne spectrometer for observing diffuse emission line targets," Proc. SPIE 8446, Ground-based and Airborne Instrumentation for Astronomy IV(84464K), (2012).

[13] W. D. Pence, [The FITS Support Office] http://fits.gsfc.nasa.gov/. 